\title{
ATTENUATION CORRECTION AND NORMALISATION FOR QUANTIFICATION OF CONTRAST ENHANCEMENT IN ULTRASOUND IMAGES OF CAROTID ARTERIES
}

${ }^{1}$ WING KEUNG CHEUNG, ${ }^{2}$ DOROTHY M. GUJRAL, ${ }^{3}$ BENOY N. SHAH, ${ }^{3}$ NAVTEJ S. CHAHAL, ${ }^{3}$ SANJEEV BHATTACHARYYA, ${ }^{4}$ DAVID O. COSGROVE, ${ }^{5}$ ROBERT J. ECKERSLEY, ${ }^{2}$ KEVIN J. HARRINGTON, ${ }^{3}$ ROXY SENIOR, ${ }^{2}$ CHRISTOPHER M. NUTTING, ${ }^{1}$ MENG-XING TANG

${ }^{1}$ Department of Bioengineering, Imperial College, Exhibition Road, London, SW7 2AZ

${ }^{2}$ Head and Neck Unit, The Royal Marsden Hospital, 203 Fulham Road, London, SW3 6JJ, UK

${ }^{3}$ Biomedical Research Unit, NHLI, Imperial College, London, UK

${ }^{4}$ Imaging Department, Imperial College London

${ }^{5}$ Division of Imaging Sciences, King's College London

Address correspondence to: Meng-Xing Tang, Department of Bioengineering, Imperial College London, London SW7 2AZ, UK. E-mail: mengxing.tang@imperial.ac.uk 


\begin{abstract}
An automated attenuation correction and normalisation algorithm was developed to improve the quantification of contrast enhancement in ultrasound images of carotid arteries. The algorithm first corrects attenuation artefact and normalises intensity within the contrast agentfilled lumen and then extends the correction and normalisation to regions beyond the lumen. The algorithm was first validated on phantoms consisting of contrast agent-filled vessels embedded in tissue-mimicking materials of known attenuation. It was subsequently applied to in vivo contrast-enhanced ultrasound (CEUS) images of human carotid arteries. Both in vitro and in vivo results indicated significant reduction in the shadowing artefact and improved homogeneity within the carotid lumens after the correction. The error in quantification of microbubble contrast enhancement caused by attenuation on phantoms was reduced from $55 \%$ to $5 \%$ on average. In conclusion, the proposed method exhibited great potential in reducing attenuation artefact and improving quantification in contrast-enhanced ultrasound of carotid arteries.
\end{abstract}

Key Words: Attenuation correction, Contrast-enhanced ultrasound, Carotid artery, Adventitial vasa vasorum, Perfusion quantification, Shadowing. 


\section{Introduction}

Stroke is a major cause of mortality, morbidity and long-term disability, resulting in a substantial economic burden on health and social services (Murray and Lopez 1997). Carotid atherosclerotic plaque is one of the major preventable causes of stroke (U-King-Im et al. 2009). Plaques at risk of rupture (vulnerable plaques) are not necessarily those that impinge most substantially upon the lumen (Topol and Nissen 1995). Nevertheless, current clinical imaging investigations still focus on quantifying the degree of luminal stenosis and, hence, are relatively poor at predicting which patients will suffer a stroke. Recent studies have identified plaque neovascularisation as being a key feature of vulnerable plaques (Hellings et al. 2010; Virmani et al. 2006). Furthermore, abnormal proliferation of adventitial vasa vasorum (VV) occurs early at sites of atherosclerosis and is thought to be a precursor to atherosclerosis and an early biomarker of vascular damage (Feinstein 2006; Macioch et al. 2004). An imaging tool capable of detecting and quantifying such vascular features would offer valuable information for the diagnosis and management of this important disease.

Ultrasonography (US) is regarded as one of the most promising tools in assisting diagnosis and management of carotid artery disease because of its non-ionizing nature and real-time imaging in good spatial resolution, with relatively low cost and high accessibility. Recently, contrast-enhanced ultrasound (CEUS) has made it possible to image and quantify neovascularisation in plaques as well as adventitial VV (Feinstein 2006). However, current quantification of contrast enhancement is significantly limited by spatially heterogeneous and patient-specific attenuation (Tang et al. 2008, 2011). During a US scan, ultrasound echo from a target is affected by attenuation between the US transducer and the target. Time gain compensation (TGC) is commonly used for correcting attenuation where echo signals are amplified as a function of time, so the further the echoes come from, the higher the signal gain is. However, such TGC cannot account for spatially heterogeneous attenuation caused by either heterogeneous tissue distribution or non-uniform contact between the probe and skin. It is also difficult for TGC to account for variations in tissue attenuating properties across patient populations. Consequently, it is common to see (i) shadowing in vascular ultrasound images (e.g., see Fig. 1), a manifestation of spatially heterogeneous attenuation; and (ii) variations in image intensity between patients, a manifestation of population variation. It should be noted that attenuation may not be visually identifiable because of image compression at display, but can still cause significant errors in quantification based on image intensity, which limits the usefulness of quantification of CEUS in clinical applications. Although there have been studies on correction of attenuation in CEUS images in general (Mari et al. 2010; Mule et al. 2008; Tang et al. 2008), we are not aware of any study on CEUS images of carotid arteries where accurate quantification of plaque neovascularisation and abnormal proliferation of $\mathrm{VV}$ is valuable. 


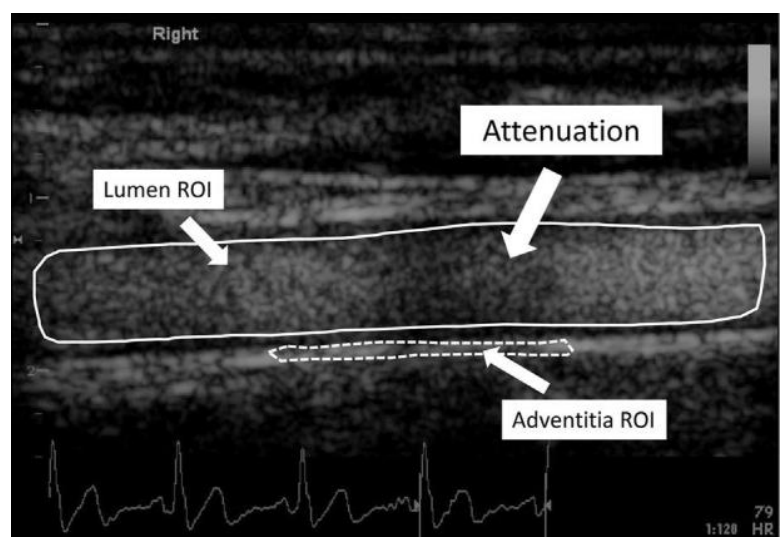

Fig. 1. Regions of interest (ROI) in the lumen (solid line) and vessel wall (dashed line).

The objective of this study was to develop an attenuation correction and normalisation technique for CEUS carotid artery imaging, to correct for spatially heterogeneous and patient-specific attenuation and thus improve the quantification of contrast enhancement in plaques and VV. This technique was initially validated on a carotid artery-mimicking phantom and then, as an initial clinical demonstration, applied to ultrasound contrast enhancement in carotid artery vessel wall in a cohort of patients.

\section{Methods}

\section{Attenuation correction and image normalisation algorithms}

An attenuation correction algorithm for CEUS carotid artery images was developed based on the assumption that microbubble contrast agents are well mixed in the lumen, and hence, the image intensity across the vessel lumen should be homogeneous (except for intensity variations caused by speckle) if attenuation is properly corrected for. Microbubbles, when injected intravenously, are expected to have been well mixed in the flow when they arrive at the carotid artery. On the basis of this assumption, the algorithm initially estimates and corrects for the attenuation within the carotid lumen and then extends the correction at the lumen boundary to the vessel wall next to the lumen. Furthermore, the images are normalised so that quantification of contrast enhancement is less affected by variations in patient dose of contrast agents.

Analysis of CEUS video sequences was performed off-line using software developed inhouse using MATLAB (The MathWorks, Natick, MA, USA). Regions of interest (ROIs) were selected manually, one to segment the lumen and the others to include regions in adventitia where quantification is required (Fig. 1). The motion of the lumen and adventitia ROIs in the video sequence was tracked and corrected by employing a piecewise block matching algorithm (Briechle and Hanebeck 2001; Golemati et al. 2003). As a result of motion correction, all images in the sequence were aligned to the first image. 
The attenuation correction algorithm consists of the following specific steps. All computations were performed on linearised video data. The video data are log compressed; linearised video data refer to the anti-log decompressed video data.

1. Based on the segmented lumen, the relative smoothed intensity profile A, which is a function of accumulated attenuation within the lumen, was estimated by adopting a low-pass Gaussian filter (Tang et al. 2008) as indicated in the equation

$$
A_{\text {lumen }}(x, y)=I_{\text {lumen }}(x, y) * G(x, y)
$$

where $G(x, y)=\frac{1}{2 \pi \sigma^{2}} \cdot e^{-\frac{x^{2}+y^{2}}{2 \sigma^{2}}}, x$ and $y$ are spatial coordinates, $*$ denotes convolution

The choice of $\sigma$, the standard deviation of the Gaussian kernel, depends on the imaging settings. Here the standard deviation is set to be at least twice the speckle size measured on the image for both phantom and in vivo studies. Such a filter diminishes the speckle and leaves the spatially smoothed intensity profile. One issue with the filtering is that at the boundary of the lumen, the filter covers pixels outside the lumen. To resolve this issue, a mirror image approach was adopted; that is, the pixels outside the bounds of the lumen were computed by mirror reflecting the lumen across the lumen border.

2. The attenuation correction was performed within the segmented lumen as described in the equation

$$
C I_{\text {lumen }}(x, y)=I_{\text {lumen }}(x, y) /\left(A_{\text {lumen }}(x, y)+\text { regulariser }\right)
$$

where $C I_{\text {lumen }}(x, y)$ denotes corrected image within the lumen.

The regulariser is a constant that avoids the over-amplification of noise when $A_{\text {lumen }}$ in the denominator in eqn (2) becomes close to zero. It plays a crucial role in this correction process, as too large a regulariser would leave the image uncorrected, and too small a regulariser would cause overcompensation because of noise. In this study, the value of the regulariser is set at 0.08 for the phantom study and 0.1 for the in vivo study. These values are obtained by minimising the normalised intensity fluctuation (NIF) within the corrected lumen defined as

$$
N I F=\frac{\sqrt{\sum(C I(x, y)-\langle C I(x, y)\rangle)^{2}}}{\langle C I(x, y)\rangle}
$$

where $C I$ is the intensity of pixel at position $(\mathrm{x}, \mathrm{y})$, and $\langle C I(x, y)\rangle$ is the spatial average intensity within corrected lumen. The variation of this index as a function of the regulariser for the phantom study is illustrated in Figure 2. 


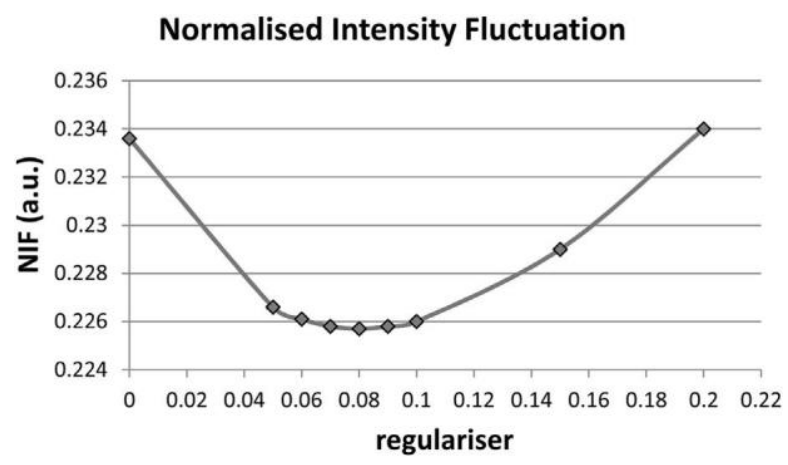

Fig. 2. Normalised intensity fluctuation (NIF) within a corrected lumen.

3. Based on the estimated $C_{\text {lumen }}(x, y)$, which covers the whole lumen, the correction factors at the lower $\left(A_{l b}\right)$ and upper $\left(A_{u b}\right)$ boundaries of the lumen were extended to regions below $\left(I_{l r}\right)$ and above the lumen $\left(I_{u r}\right)$, including the adventitia, using

$$
\begin{gathered}
C I_{u r}(x, y)=I_{u r}(x, y) /\left(A_{u b}(x, y)+\text { regulariser }\right) \\
C I_{l r}(x, y)=I_{l r}(x, y) /\left(A_{l b}(x, y)+\text { regulariser }\right)
\end{gathered}
$$

\section{Image normalisation}

The image is then normalised by the mode intensity within the lumen. After normalisation, the peak in the lumen intensity histogram is located at intensity equal to one. This is to reduce the variations in CEUS quantification caused by variations in both tissue attenuation properties and contrast concentration in different patients.

\section{Evaluation of lumen intensity homogeneity}

As a first step in evaluation of the attenuation correction algorithm, image intensity histograms were generated to characterise the change in intensity homogeneity within the lumen before and after the correction. The full width at half-maximum (FWHM) of the peak in the lumen intensity histogram is calculated as a measure of lumen intensity homogeneity.

\section{Phantom setup and validation}

The attenuation correction algorithm was first validated on a carotid artery-mimicking phantom constructed in-house and illustrated in Figure 3. It consisted of two contrast agentfilled vessels embedded in a tissue-mimicking phantom (TMM2) (Madsen et al. 1998). The first vessel simulates the carotid artery, and the second is $1 \mathrm{~cm}$ below the first, representing 
target regions containing microbubble contrast agents whose concentration needs to be quantified. An additional attenuating material (TMM1) was placed under the probe and covering half of the TMM2, causing additional attenuation to only half of the phantom. Although the function of the upper vessel was to mimic the carotid artery, the function of the lower vessel was to create a region with controlled bubble concentration. Given that the microbubbles were well mixed and the TMMs under the tube are homogeneous, such a setup offers both measurements with attenuation (left-hand side [LHS]) and control measurements (right-hand side [RHS]) within the same image acquisition, and any extra signal loss on the LHS of the phantom compared with the RHS is due to the attenuation caused by the additional attenuation material.

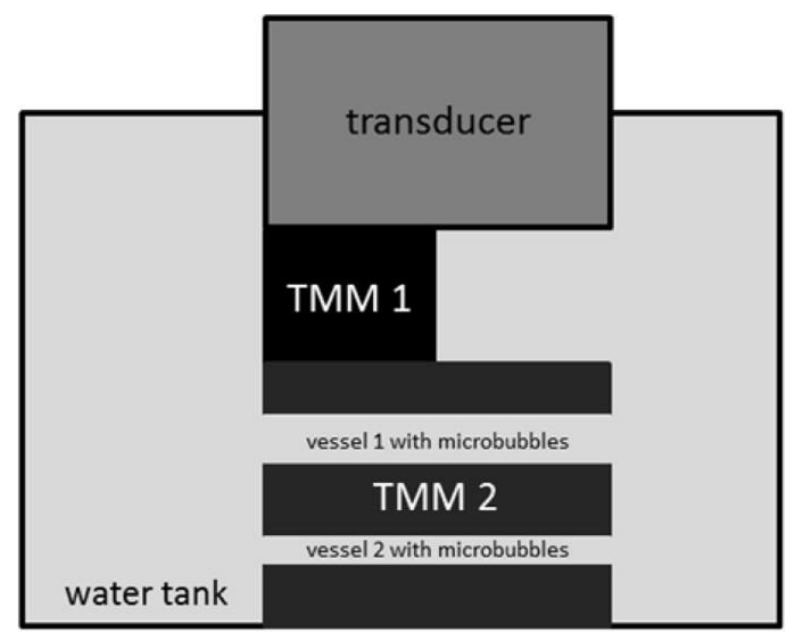

Fig. 3. Carotid artery-mimicking setup with additional attenuation material (TMM1) on the left-hand side of the phantom. TMM = tissue-mimicking material.

TMM1 and TMM2 were constructed according to the protocol of Madsen et al. (1998). The estimated attenuation of TMM1 or TMM2 was $0.5 \mathrm{~dB} \mathrm{~cm} 21 \mathrm{MHz}-1$. Homemade lipid shell microbubbles were used at two concentrations: $7.50 \times 10^{6} / \mathrm{mL}$ and $3.75 \times 10^{6} / \mathrm{mL}$. An AplioXG scanner (PLT-704 SBT, 4- to 11-MHz linear probe, Toshiba, Tokyo, Japan) was used to scan the phantom with the following settings: mechanical index $=0.1$, gain $=70$, dynamic range $=80, \mathrm{TGC}=$ manually adjusted, frequency $=4 / 8 \mathrm{MHz}$. Two groups of ROIs (bubble regions within the lower vessel and tissue regions below that vessel) were selected on both the attenuated and control sides of the phantom (Fig. 4), and the average intensities before and after attenuation correction were compared. The locations of ROIs are indicated in Figure 4, including regions for attenuated bubble signals, unattenuated bubble signals, attenuated tissue signals and unattenuated tissue signals.

\section{Clinical application (vessel wall)}

Forty-eight patients previously treated for head and neck cancer (HNC) with at least one risk factor for atherosclerosis were recruited from a cancer centre. The study was approved by the institutional research and ethics committee, and each patient provided informed consent. 
CEUS image sequences were acquired on both sides of the neck with a clinical scanner (GE Vivid 7 with a 9-MHz broadband linear array transducer). A GE scanner (Vivid 7, linear array 9L transducer, GE Healthcare) was used to scan the phantom with the following settings: mechanical index $=0.21$, gain $=0$, dynamic range $=54, \mathrm{TGC}=$ manually adjusted, frequency $=3.2 / 6.4 \mathrm{MHz}$. Contrast-enhanced ultrasound video loops were taken using a commercially available ultrasound contrast agent, SonoVue (Bracco, Milan, Italy), given as an intravenous infusion via a peripheral vein at the rate of $1.2 \mathrm{~mL} / \mathrm{min}$. The infusion was delivered over a total of 5-7 min. Imaging was performed in real time before arrival of and after saturation of the carotid artery with SonoVue.

The FWHM of lumen intensity histogram for both uncorrected and corrected images was calculated.

\section{Statistical analysis}

The FWHM of lumen intensity histograms across the patient population are presented as means and standard deviations (SD). The FWHM of lumen intensity histograms of uncorrected and corrected images were compared using paired-sample $t$-tests. A two-tailed test was used, with $\alpha$ set at 0.05 . Statistical analyses were performed using on-line Prism 6 software (GraphPad, La Jolla, CA, USA).

\section{Results}

\section{Phantom validation}

Images of the phantom before and after correction are provided in Figure 4. The image intensity in the lumen on the LHS is significantly attenuated before correction and is restored to a level similar to that on the RHS after correction. The attenuated tissue region under the vessel on the LHS has also been compensated. Note that the difference in speed of sound between the additional attenuation material and water caused some slight image distortion for the phantom. 
a

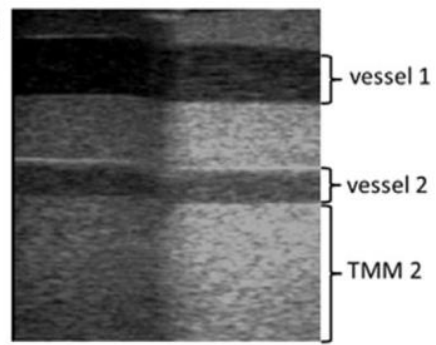

high concentration, B-mode

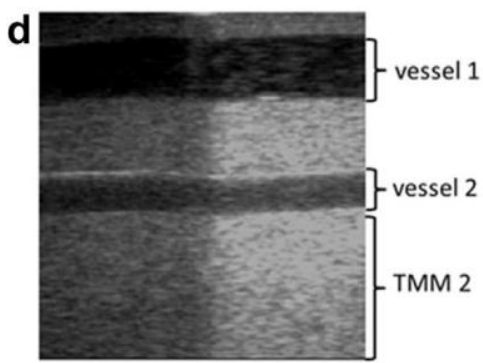

low concentration, B-mode b

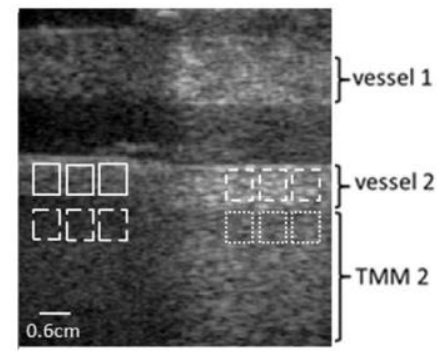

high concentration, uncorrected

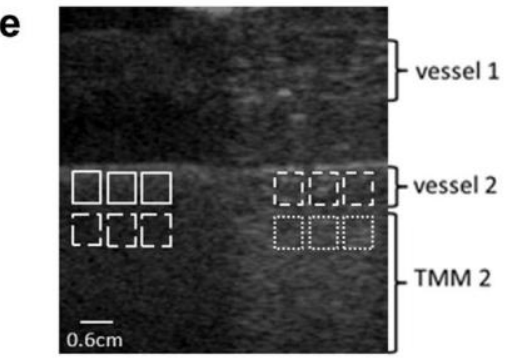

low concentration, uncorrected

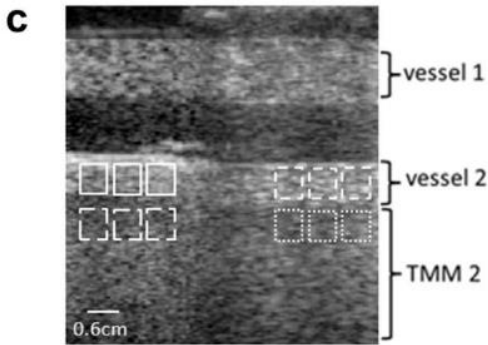

high concentration, corrected

f

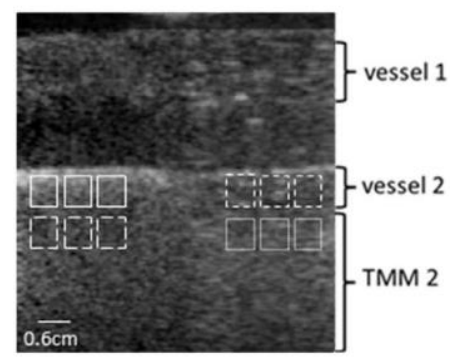

low concentration, corrected

Fig. 4. Contrast-enhanced ultrasound images of a carotid artery-mimicking phantom before and after attenuation correction with B-mode images as reference. Regions of interest (ROIs) with solid outlines represent regions of attenuated bubble signals (bubble attenuated), ROIs with dashed outlines represent regions of unattenuated bubble signals (bubble control), ROIs with long-dashed lines represent regions of attenuated tissue signals (tissue attenuated) and ROIs with round dotted lines represent regions of unattenuated tissue signals (tissue control). $\mathrm{TMM}=$ tissue-mimicking material.

Table 1 (A, B) summarizes the ROI measurements of the phantom with high bubble concentration before and after attenuation correction. It can be seen that the intensity error for quantification of microbubble signals was reduced from $61 \%$ to $7 \%$ after correction. 
Table 1A Bubble ROIs measurement of phantom before and after attenuation correction (high concentration)

\begin{tabular}{|c|c|c|c|}
\hline & $\begin{array}{c}\text { ROI (bubble - } \\
\text { control) }\end{array}$ & $\begin{array}{l}\text { ROI (bubble - } \\
\text { attenuated) uncorrected }\end{array}$ & $\begin{array}{l}\text { ROI (bubble - } \\
\text { attenuated) corrected }\end{array}$ \\
\hline $\begin{array}{c}\text { Intensity } \\
(\text { mean } \pm \mathrm{SD})\end{array}$ & $1.11 \pm 0.053$ & $0.439 \pm 0.058$ & $1.03 \pm 0.029$ \\
\hline $\begin{array}{l}\text { Difference } \\
\text { to control }\end{array}$ & & $61 \% \pm 9 \%$ & $7 \% \pm 9 \%$ \\
\hline
\end{tabular}

Table 1B Tissue ROIs measurement of phantom before and after attenuation correction (high concentration)

\begin{tabular}{|c|c|c|c|}
\hline & $\begin{array}{c}\text { ROI (tissue - } \\
\text { control) }\end{array}$ & $\begin{array}{c}\text { ROI (tissue - attenuated) } \\
\text { uncorrected }\end{array}$ & $\begin{array}{c}\text { ROI (tissue - } \\
\text { attenuated) corrected }\end{array}$ \\
\hline Intensity & $0.670 \pm 0.018$ & $0.283 \pm 0.017$ & $0.600 \pm 0.033$ \\
\hline Difference & & $58 \% \pm 2 \%$ & $10 \% \pm 4 \%$ \\
\hline to control & & & \\
\hline
\end{tabular}

Table 2 (A, B) summarizes the ROI measurements of the phantom with low bubble concentration before and after attenuation correction. It can be seen that the intensity error for quantification of microbubble signals was reduced from $48 \%$ to $2 \%$ after correction. 
Table 2A Bubble ROIs measurement of phantom before and after attenuation correction (low concentration)

\begin{tabular}{|c|c|c|c|}
\hline & $\begin{array}{c}\text { ROI (bubble - } \\
\text { control) }\end{array}$ & $\begin{array}{c}\text { ROI (bubble - } \\
\text { attenuated) uncorrected }\end{array}$ & $\begin{array}{c}\text { ROI (bubble - } \\
\text { attenuated) corrected }\end{array}$ \\
\hline Intensity & $0.819 \pm 0.103$ & $0.429 \pm 0.026$ & $0.838 \pm 0.047$ \\
\hline Difference & & $48 \% \pm 6 \%$ & $2 \% \pm 9 \%$ \\
\hline to control & & & \\
\hline
\end{tabular}

Table 2B Tissue ROIs measurement of phantom before and after attenuation correction (low concentration)

\begin{tabular}{|c|c|c|c|}
\hline & $\begin{array}{c}\text { ROI (tissue - } \\
\text { control) }\end{array}$ & $\begin{array}{c}\text { ROI (tissue - attenuated) } \\
\text { uncorrected }\end{array}$ & $\begin{array}{c}\text { ROI (tissue - attenuated) } \\
\text { corrected }\end{array}$ \\
\hline Intensity & $0.683 \pm 0.008$ & $0.275 \pm 0.012$ & $0.538 \pm 0.044$ \\
\hline Difference & & $60 \% \pm 6 \%$ & $21 \% \pm 9 \%$ \\
\hline to control & & & \\
\hline
\end{tabular}

In vivo results

Contrast-enhanced ultrasound images of carotid arteries before and after attenuation correction are provided in Figure 5. It can be seen that in both cases, part of the lumen is darker than the rest before the correction, and such attenuation artefacts are corrected for after applying the developed algorithms. Visually, the shadowing in the image was removed. In addition, the visualization of contrast enhancement in the vessel wall was improved. 

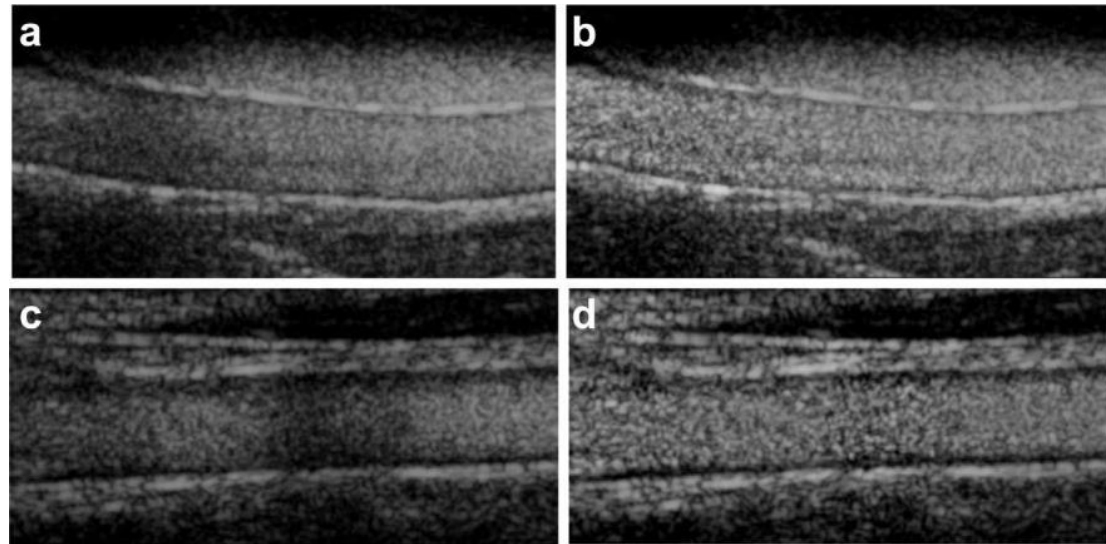

Fig. 5. Examples of contrast-enhanced ultrasound images of carotid arteries before and after attenuation correction. Top row: Patient A before (a) and after (b) attenuation correction. Bottom row: Patient B before (c) and after (d) attenuation correction.

\section{Histogram of lumen intensity and FWHM evaluation}

Lumen intensity histograms for the two patients are illustrated in Figure 6. The FWHM of the intensity histogram within the lumen was reduced from 0.350 (Fig. 6a) to 0.195 (Fig. 6b) after attenuation correction for patient $\mathrm{A}$ and from 0.500 (Fig. 6c) to 0.150 (Fig. 6d) for patient B.
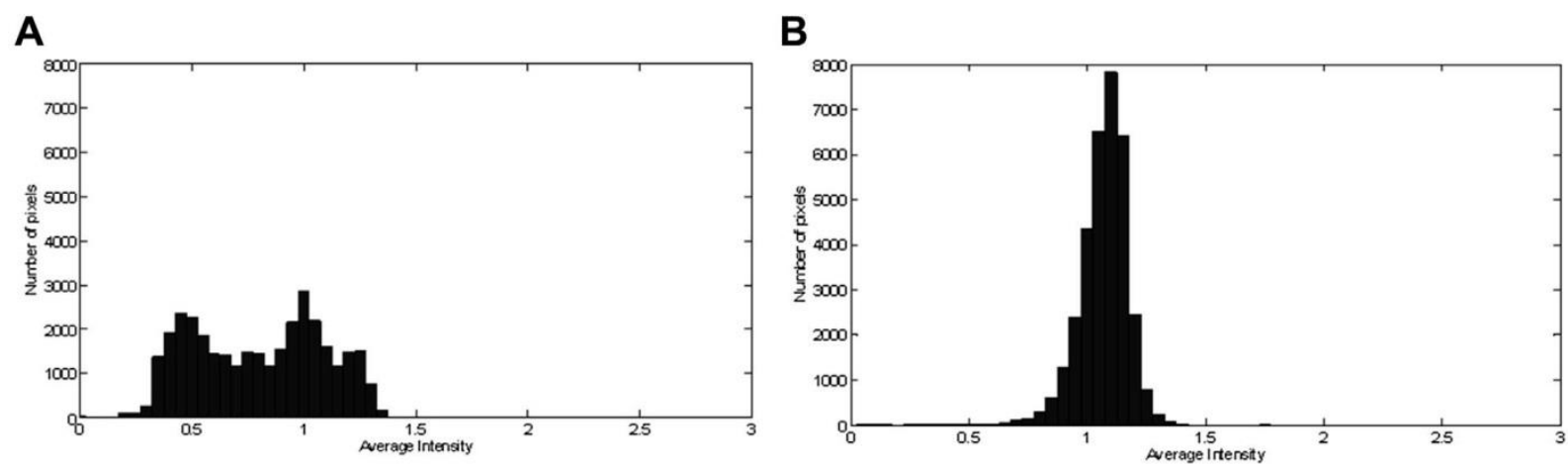

C

\section{D}
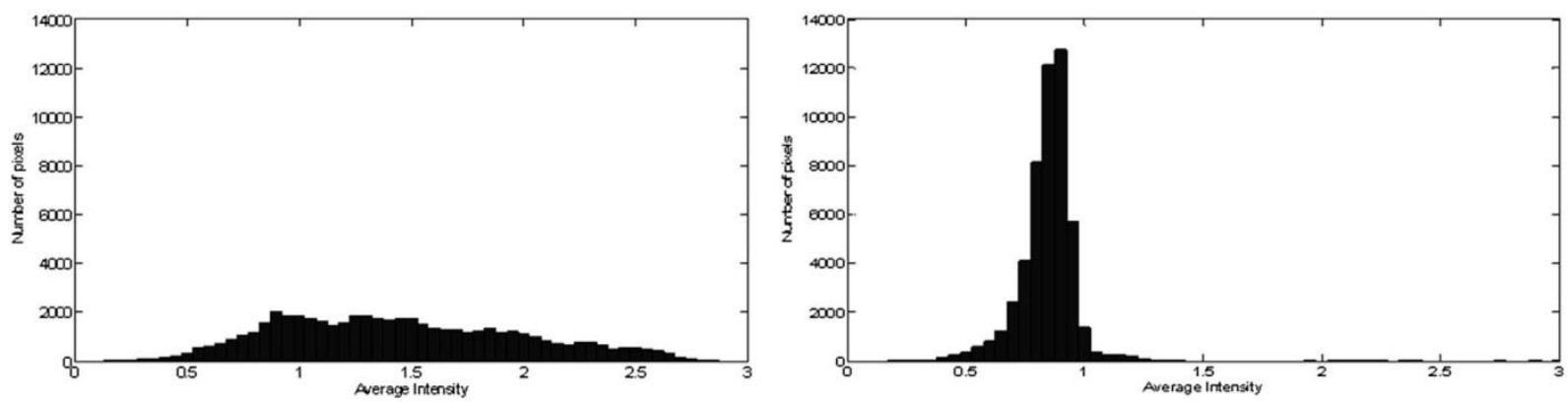

Fig. 6. Image intensity within lumen before and after attenuation correction. Top row: Patient A before (a) and after (b) attenuation correction. Bottom row: Patient B before (c) and after (d) attenuation correction. 
The FWHMs of lumen intensity histogram for all 48 patients were compared before and after attenuation correction. The mean \pm SD of the FWHM was $0.346 \pm 0.138$ before and $0.135 \pm$ 0.038 after the correction, representing a 61.0\% decrease in FWHM with the corrected image compared with the uncorrected one. This decrease in FWHM is statistically significant (paired $t$-test, $p<0.0001$ ).

\section{Discussion}

An attenuation correction and image normalisation method for CEUS carotid artery images has been developed for more reliable quantification of contrast enhancement. The method uses the lumen as a reference, where the image brightness is assumed to be homogeneous if attenuation is properly corrected. Our initial results on phantoms and in vivo carotid arteries indicate that the correction method significantly reduces attenuation artefact. On phantom evaluation, the error in quantifying microbubble contrast enhancement caused by attenuation is reduced from $48 \%-61 \%$ to $2 \%-7 \%$. For in vivo data, the correction improved the lumen homogeneity, measured as FWHM of the lumen intensity histogram, by $61 \%$ on average.

The method assumes that microbubbles are well mixed across the lumen and, therefore, that image bright-ness should be homogeneous if attenuation is properly corrected. This is a reasonable assumption, as the micro-bubbles are injected as an intravenous infusion, and by the time they get to the carotid artery they would have been well mixed in blood.

The correction algorithm includes two parameters: the Gaussian spatial low-pass filter size and the regulariser. These are independent of each other. The filter size (sigma) is determined by the size of the speckle (it needs to be large enough to remove the speckle), whereas the regulariser is determined by the system noise level.

There are a range of imaging variables including ultrasound scanner settings such as frequency, mechanical index, various gain settings and dynamic range and the type of scan format (linear or sector). The proposed algorithms are not expected to be significantly affected by the variations in most of these factors in general, as they do not violate the model assumption: microbubbles within vessel lumen are well mixed and hence vessel lumen signals should be homogeneous. However, some factors, such as dynamic range, if set too low, could cause image saturation and hence affect the results.

To illustrate the effect of attenuation in both the axial and lateral directions, we have performed correction in the $\mathrm{x}$-direction alone by averaging the estimated attenuation 
vertically and compared with the correction in both directions. From Figure 7d, it can be seen that the attenuation in both directions is significant and should be corrected.
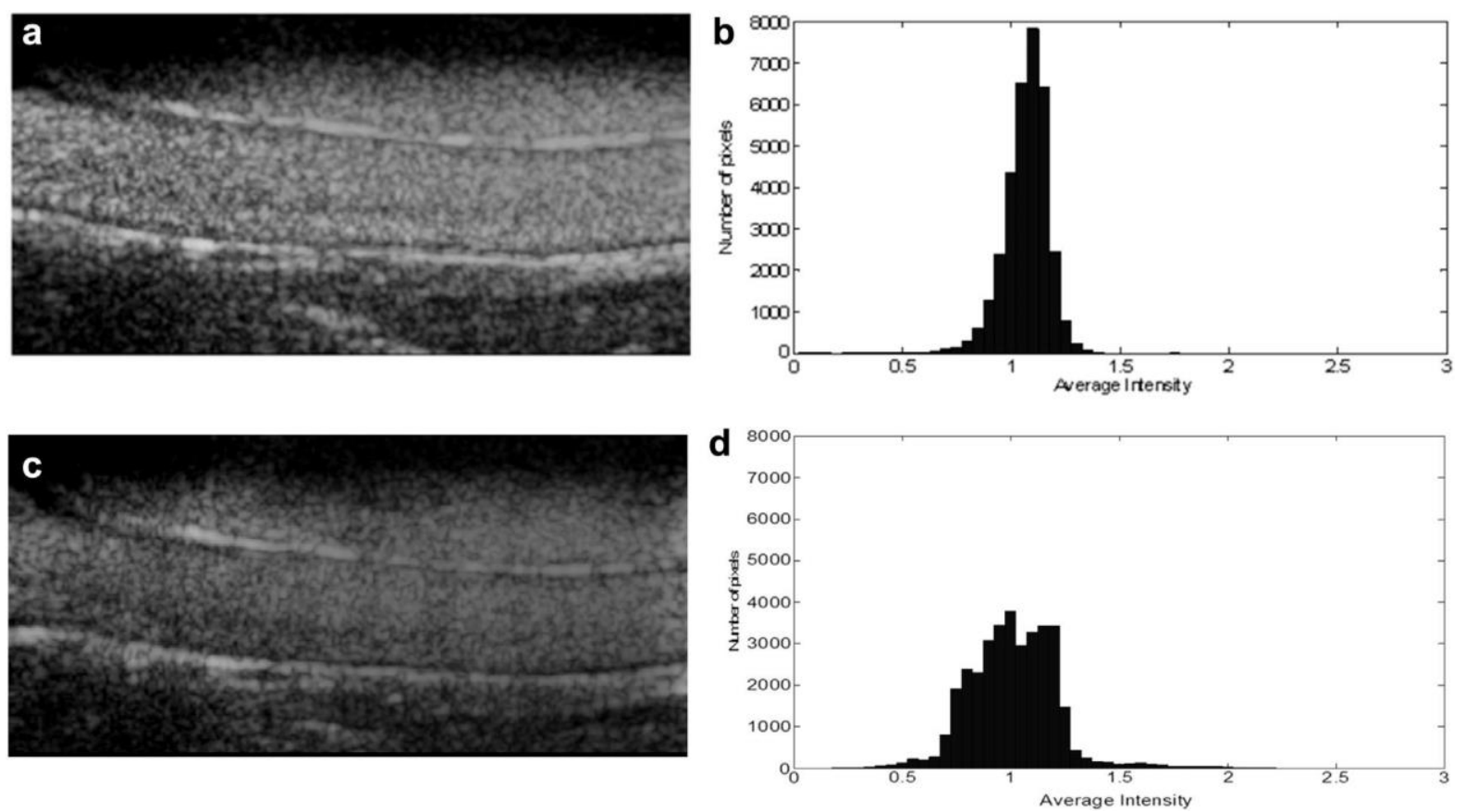

Fig. 7. Top row: Attenuation correction in both $\mathrm{x}$ - and y-directions-(a) contrast-enhanced ultrasound image and (b) image intensity histogram within lumen after attenuation correction. Bottom row: Attenuation correction in x-direction only-(c) contrast-enhanced ultrasound image and (d) image intensity histogram within lumen after attenuation correction.

Besides attenuation, non-linear imaging artefacts (Tang and Eckersley 2006; Tang et al. 2010) also commonly exist in CEUS images. Such artefacts originate from the assumption of totally linear ultrasound transmission and propagation, on one hand, and the non-linear propagation of the transmitted ultrasound pulse through tissue and/or microbubble clouds in reality, on the other hand (Tang and Eckersley 2006; Tang et al. 2010). These artefacts vary with both ultrasound amplitude and frequency. Clinical examples of such artefacts have recently been reported (Ten Kate et al. 2012; Van den Oord et al. 2013). In the present study, the quantification results are likely to contain, besides desirable neovascularisation and VV signals, significant undesirable non-linear artefacts. It is important to address both attenuation and the non-linear artefacts to achieve reliable quantification in CEUS images. We are currently working on correction of non-linear artefacts, and initial results have already been reported (Yildiz et al. 2015) with the aim of incorporating this into our software. Currently, further use of our software in a clinical setting would be suitable for quantifying differences in contrast intensity between patients and controls, where there is a reasonable expectation that non-linear imaging artefacts would be similar for both groups.

Attenuation artefacts in CEUS images, together with other factors, have made reliable quantification difficult and have led to diagnostic uncertainty. This is the first study, as far as 
we are aware, to develop attenuation and normalisation techniques for CEUS carotid images. It does not require modification to any imaging system hardware and can be packaged as a software module and integrated into existing commercial ultrasound scanners. Although in this work the correction of attenuation and normalisation were conducted offline, real-time processing is feasible, as the algorithm involves only simple filtering and division. Currently the more time-consuming part is the manual lumen segmentation and motion tracking, but given that the algorithm does not depend on accurate lumen segmentation and tracking, it is feasible for these to be done in real time. This technique has potential to be extended to other views of carotid arteries, and this requires further studies. In addition, quantification of neovascularisation may be tested in a clinical setting as a possible early surrogate biomarker of atherosclerosis against validated surrogates of stroke risk such as intima-media thickness to determine if there is any clinical correlation. The use of controls would be valuable in determining changes related specifically to signal intensity caused by neovascularisation, both in the adventitia and in plaques.

\section{Conclusions}

Our attenuation correction and normalisation method resulted in a significant reduction of attenuation artefacts both on a carotid artery-mimicking phantom and in vivo. This represents a step toward reliable quantification of contrast enhancement in CEUS carotid images.

\section{Acknowledgments}

Meng-Xing Tang acknowledges the funding from EPSRC (EP/K503733/1) and Bagrit Foundation. DMG, KJH and CMN acknowledge support from the RM/ICR NIHR Biomedical Research Centre and Cancer Research UK Programme Grant C7224/ A13407. 


\section{References}

Briechle K, Hanebeck UD. Template matching using fast normalized cross correlation. Proc SPIE Opt Pattern Recog XII 2001;4387:95-102.

Feinstein SB. Contrast ultrasound imaging of the carotid artery vasa vasorum and atherosclerotic plaque neovascularization. J Am Coll Cardiol 2006;48:236-243.

Golemati S, Sassano A, Lever MJ, Bharath AA, Dhanjil S, Nicolaides AN. Carotid artery wall motion estimated from B- mode ultrasound using region tracking and block matching. Ultra- sound Med Biol 2003;29:387-399.

Hellings WE, Peeters W, Moll FL, Piers SRD, van Setten J, Van der Spek PJ, de Vries JP, Seldenrijk KA, De Bruin PC, Vink A, Velema E, de Kleijn DPV, Pasterkamp G. Composition of carotid atherosclerotic plaque is associated with cardiovascular outcome: A prognostic study. Circulation 2010;121:1941-1950.

Macioch JE, Katsamakis CD, Robin J, Liebson PR, Meyer PM, Geohas C, Raichlen JS, Davidson MH, Feinstein SB. Effect of contrast enhancement on measurement of carotid artery intimal medial thickness. Vasc Med 2004;9:7-12.

Madsen EL, Frank GR, Dong F. Liquid or solid ultrasonically tissue-mimicking materials with very low scatter. Ultrasound Med Biol 1998;24:535-542.

Mari JM, Hibbs K, Stride E, Eckersley RJ, Tang MX. An approximate nonlinear model for time gain compensation of amplitude modu- lated images of ultrasound contrast agent perfusion. IEEE Trans Ul- trason Ferroelectr Freq Control 2010;57:818-829.

Mule S, De Cesare A, Lucidarme O, Frouin F, Herment A. Regularized estimation of contrast agent attenuation to improve the imaging of microbubbles in small animal studies. Ultrasound Med Biol 2008; 34:938-948.

Murray CJ, Lopez AD. Mortality by cause for eight regions of the world: Global Burden of Disease Study. Lancet 1997;349:1269-1276. 
Tang MX, Eckersley RJ. Nonlinear propagation of ultrasound through microbubble contrast agents and implications for imaging. IEEE Trans Ultrason Ferroelectr Freq Control 2006;53:2406-2415.

Tang MX, Kamiyama N, Eckersley RJ. Effects of nonlinear propagation in ultrasound contrast agent imaging. Ultrasound Med Biol 2010;36: 459-466.

Tang MX, Mari JM, Wells PNT, Eckersley RJ. Attenuation correction in ultrasound contrast agent imaging: Elementary theory and prelimi- nary experimental evaluation. Ultrasound Med Biol 2008;34: 1998-2008.

Tang MX, Mulvana H, Gauthier T, Lim AKP, Cosgrove DO, Eckersley RJ, Stride E. Quantitative contrast-enhanced ultrasound imaging: A review of sources of variability. Interface Focus 2011; 1:520-539.

Ten Kate GL, Renaud GGJ, Akkus Z, van den Oord SCH, ten Cate FJ, Shamdasani V, Entrekin RR, Sijbrands EJG, de Jong N, Bosch JG, Schinkel AFL, van der Steen AFW. Farwall pseudoenhancement during contrast-enhanced ultrasound of the carotid arteries: Clinical description and in vitro reproduction. Ultrasound Med Biol 2012;38: 593-600.

Topol EJ, Nissen SE. Our preoccupation with coronary luminology: The dissociation between clinical and angiographic findings in ischemic- heart-disease. Circulation 1995;92:2333-2342.

U-King-Im JM, Young V, Gillard JH. Carotid-artery imaging in the diagnosis and management of patients at risk of stroke. Lancet Neurol 2009;8:569-580.

Van den Oord SC, Renaud G, Bosch JG, de Jong N, van der Steen AF, Schinkel AF. Far wall pseudo-enhancement: A neglected artifact in carotid contrast-enhanced ultrasound? Atherosclerosis 2013; 229:451-452. 
Virmani R, Burke AP, Farb A, Kolodgie FD. Pathology of the vulnerable plaque. J Am Coll Cardiol 2006;47:C13-C18.

Yildiz Y, Eckersley RJ, Senior R, Lim A, Cosgrove DO, Tang MX. Correction of nonlinear propagation artifact in contrast enhanced ultrasound imaging of carotid arteries: methods and in vitro evaluation. Ultrasound Med Biol 2015 (in press). 